\title{
FRONTIERS
}

\section{Physics Now}

\section{Reza Mansouri}

\section{What does physics have to do with sustainable economic growth? Quite a lot, as it happens.}

$\mathbf{T}$ he North-South divide in economic development is often matched by a North-South divide in science and technology. Governments in the North and the South, as well as international donors, have at last grasped the reality that science and technology are crucial to building a nation's institutions in areas such as transport, agriculture, health, law and industry.

However, in strengthening their scientific and technological base, developing countries have tended to focus on agricultural research, biology and genetic engineering, space science, and information and communication technologies. One of the most important sciences underpinning development - physics - is barely on the economic development radar screen.

Ignoring physics is a mistake not only because of its fundamental contributions to all science, but also because national capacity in physics correlates strongly with economic performance.

An assessment of the state of physics in a country can shed light on how successfully it is developing and will develop in the future, particularly a nation's ability to make use of advanced technologies. China, for example, accounts for $3 \%$ of the world's trade in hightechnology goods and services, which is the highest percentage among developing countries. India accounts for $1 \%$ of global trade in high-technology goods and services, which is the second highest percentage among developing countries.

It should come as no surprise that these two countries also the rank first and second in the developing world in the physical sciences - as measured by various indices, including articles published in international peer-reviewed journals and patents. The fact is that most high-technology products and services - computers, scientific instruments, electrical machinery and electronics - are based on research and development in the physical sciences.

How well are developing nations doing in physics? A good measure of a nation's capability in any science is the number of articles published by its scientists in international peer-reviewed journals.

\section{VIEWPOINT I Lu Yongxiang What others can - and cannot - learn from China}

China has developed a strategy for science, technology and innovation (STI) based on its own history, culture and circumstances. For example, in the last decades of the twentieth century, we sent a large number of students abroad for education and training. Our goal was to increase the number of researchers as quickly as possible. We are also a big country that is able to invest significant sums of money in scientific infrastructure due largely to our growing economic base. The overall strategy has been to develop our scientific and

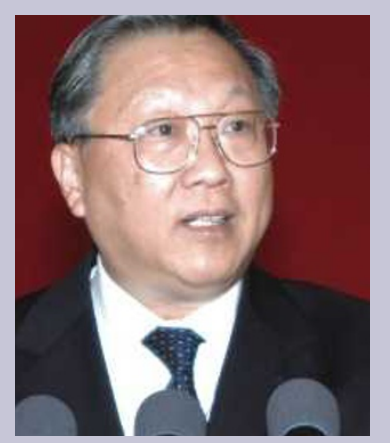
technological capabilities rapidly in order to address our most critical economic and social challenges. Yet, we also realized we had to be patient and think long term. Other developing nations have been grappling with these complex issues in their own way. As was the case with Germany, the USA and the UK, the strategies of developing countries will be based on their own historical and present-day circumstances. There are, of course, some general principles that developing nations would be wise to consider - for example, the indispensable role of government, the importance of sustained investments in education at all levels, the value of linking investments in STI to national plans for economic development, and the need to encourage private-sector investments in research and development. But each nation must determine on its own both how to pursue science-based sustainable development and the pace at which to proceed. That does not mean developing countries should not exchange ideas and learn from one another, but rather that there are many pathways to success.

Lu Yongxiang (TWAS Fellow 1990) is the president of the Chinese Academy of Sciences in Beijing, China.
In 2006, physicists in the developed world authored over $80 \%$ of the physics papers published in international peer-reviewed journals. Physicists in the USA alone were responsible for $18 \%$ of the total $(32,000$ articles).

Meanwhile, physicists in developing countries, which are home to $80 \%$ of the world's population, authored $20 \%$ of physics papers. When you consider that three developing countries - China, India and Brazil - are each responsible for approximately $1 \%$ of the total published every year, the situation looks a lot more dismal for other developing nations. Indeed, only 28 of them publish more than 100 physics papers a year in international journals. The contribution of the remainder, some 120 countries in total, is so small that it is not statistically significant. These include many of the 57 member states of the Organization of the Islamic Conference (OIC). The OIC states are home to some 1.5 billion people. Yet, physicists in OIC countries are collectively responsible for just $3 \%$ of the peer-reviewed articles published in international journals.

Governments and scientists from developing nations who are seeking a way out of this hole could look to China, which has increased its contribution to the worldwide total of peer-reviewed physics papers from about $4 \%$ to just over $14 \%$ in the past decade. Are there lessons here for other developing nations?

One lesson is that China and the five other developing countries that are most advanced in physics - Brazil, India, Iran, Mexico and Turkey - all focus on the same three subfields of the discipline: condensed matter physics, followed by optics and nuclear physics. Another lesson can be found in the development of scientific hardware. For example, China is home to state-of-the-art physics instrumentation. This has helped the country to transform its capacity in physics into technology products and services, which have helped to fuel the nation's growth.

The message is clear for all countries that are seeking to grow, and for all donors that are seeking to help them. Recent history shows that a nation's chances of technological development dim appreciably without a broad-based capacity in physics, and that without technological capability, sustainable economic growth often remains a faint hope. .

Reza Mansouri (TWAS Fellow 2000) is a professor of physics at Sharif University of Technology in Tehran, Iran. 